\title{
PROPUESTA DE UN MODELO DE PROGRAMACIÓN PARA LA DISTRIBUCIÓN DE COMBUSTIBLES, HACIA UN TERMIINAL MARÍTIMO
}

\author{
PROPOSAL OF A PROGRAMMING MODEL FOR THE DISTRIBUTION \\ OF FUELS, TOWARDS TO THE MARITIME TERMINAL
}

\author{
Roberto M. Campos Troncoso ${ }^{1}$, Jaime Sandoval Velásquez ${ }^{2}$, Miguel A. Bustamante U., ${ }^{* 2}$ \\ 1 Escuela de Auditoría e Ingeniería en Control de Gestión, Facultad de Economía y Negocios, Universidad de Talca. Talca, Chile, Dos Norte 685, 3465548 \\ Talca, Chile. rcampos@utalca.cl \\ 1 Escuela de Ingeniería Comercial, Facultad de Economía y Negocios, Universidad de Talca. Talca, Chile, Dos Norte 685, 3465548 Talca, Chile. mabu@ \\ utalca.cl \\ 2 Departamento operaciones, División programación y terminales, Empresa Nacional del Petróleo, ENAP, Calle la Marina 655, San Vicente, Talcahuano, \\ Chile. jsandovalv@enap.cl
}

El presente trabajo tiene como propósito optimizar la secuencia de productos y programación en la distribución de poliducto de combustibles, desde una refinería de petróleo hacia un terminal marítimo, así como determinar algunas alternativas eficientes. Se utilizó un modelo de redes, una programación de distribución de productos y secuencia de inyección a la línea, mediante el software de optimización. Además se determinaron volúmenes a inyectar a la línea en la mezcla de interfase entre productos adyacentes. Se encuentra una mejor solución considerando su aplicación en diferentes condiciones de variación de demanda y cantidad de productos. En relación a las alternativas se puede concluir que la evaluación de la alternativa de entregar los productos agrupando dos clientes, enviando así lotes de productos demandados por los respectivos clientes, en este caso el ahorro de 96.286 dólares, solo en un ciclo de envío a los 4 clientes, según la demanda establecida para el ejemplo planteado. Por lo tanto las circunstancias de ahorro, están condicionadas si realmente los clientes están dispuestos a postergar, o más bien esperar en el proceso de entrega de sus productos por un pago asociado a esta espera, como una penalización de entrega a la refinería.

PALABRAS CLAVE: Interface, Programar, Optimización, Refinería, Poliducto.
The purpose of this paper the optimization of the product sequence and programming in the distribution of fuel polyducts, from an oil refinery to a marine terminal, as well as to determine some efficient alternatives. We used a network model, a product distribution schedule and an injection sequence to the line, using the optimization software. In addition, volumes to be injected into the line were determined in the interphase mixture between adjacent products. A better solution is found considering its application in different conditions of variation of demand and quantity of products. In relation to the alternatives it can be concluded that the evaluation of the alternative of delivering the products grouping two customers, thus sending batches of products demanded by the respective customers, in this case the saving of $\$ 96,286$, only in one cycle of shipment to the 4 customers, according to the demand established for the proposed example. Therefore the saving circumstances are conditioned if the customers are really willing to postpone, or rather wait in the process of delivery of their products for a payment associated with this wait, such as a delivery penalty to the refinery.

KEYWORDS: Interfase, Schedule,Optimization, Refinery, Pipe line. 


\section{Introducción}

En nuestros días, una programación de distribución que satisfaga requerimientos en términos de clientes y tiempos es un desafío que se expresa como “... operaciones de un poliducto", ejecutadas en contextos determinados y ha sido calificada como "una tarea sumamente compleja” (Cafaro, 2009, pág. 5). Las primeras técnicas utilizan medios de suministro que implican "coordinar las actividades de abastecimiento" hasta las de "mercadeo" (Cafaro, 2009, pág. 26). Es así como, "en la actualidad, las compañías han descubierto que la buena administración de las operaciones y el suministro es esencial para su éxito. Un Dólar o un Euro ahorrados en la forma en que se produce o distribuye un producto representa directamente un Dólar o un Euro extra de utilidad” (Chase, Jacobs, \& Aquilano, 2009, pág. 4)

En este ambito, relevante es "la cadena de suministro como sistema de las empresas para transformar materia prima en bienes intermedios, luego en productos terminados, y finalmente entregarlos a los clientes" (Vinajera-Zamora, Maruro-Delgado, \& Ruiz-Morales, 2017, pág. 2). Surge así el concepto de cadena de suministro el cual ha adquirido importancia por cuanto permite la satisfacción de las necesidades del clientes (Arango, Zapata, \& Gomez, 2010), en una cadena de suministro (CS) (De la Hoz, Vélez, \& Lopez, 2017), la cual integra todas estas actividades con el objeto de alcanzar ventajas competitivas sustentables (Peña, Urueña, \& González, 2016).

La CS es una red de organizaciones, es a través de la cuales fluyen materiales (Peña, Urueña, \& González, 2016) y generar valor a la empresa la cual debe gestionarse de forma eficiente (Toro, Dominguez \& Zuluaga, 2016) considerando los sistemas logísticos, el tipo de empresas, productos y canales de distribución (Orjuela-Castro, Suarez-Camelo, \& Chinchilla-Ospin, 2016) convirtiendo ésta en una actividad estratégica (Gamez-Albón, Mejía-Argueta, \& León Espinoza de los Monteros, 2017).

Por una parte, el mejor proceso depende de factores como el volumen, el costo y la velocidad de transporte. (Chase, Jacobs, \& Aquilano, 2009) así como de las ventanas en las fechas de entrega (Ferrer, Cones, \& De los Santos, 2004), lo que requiere de esfuerzos de integración (Feitó, Cespón, \& Rubio, 2016) de la secuencia de productos considerando índices de "minimización de la tardanza total" (Gallardo, 2011, pág. 76), a través de un poliducto simple (Crane \& Colab, 1999) que minimice faltas de stock (Cafaro, 2009, pág. 37) atendiendo "en el ámbito de la Logística" (Almada, 2017, pág. 15) la programación de requerimientos y la elaboración (Garcia, 2007).

En este contexto, el sistema de ductos, es un medio de transporte de bajo costo con bajo consumo de energía, seguro y automatizable de alta confiabilidad (Noguera, Robles \& Guillot, 2015) pero que requiere de "supervisión y monitoreo automático" (Torres, Verde, Carrera, \& Cayetano, 2014) de la secuencia de inyección (Taha, 2012, pág. 395), "las especificaciones"(Garcia, 2007, pág. 46) y que termina con el análisis e interpretación de resultados (López, Castro, \& Guerra, 2017).

Sobre la base de lo presentado, el presente trabajo tiene como propósito analizar la optimización de la secuencia de productos y programación en la distribución de poliducto de combustibles, encontrar alternativas de envio. Desde una refinería de petróleo de una empresa ubicada en la ciudad de Concepción - Chile hacia un terminal marítimo de San Vicente región del BioBio.

\section{Metodología}

Se aplicó un modelo matemático de Programación Lineal que contiene los parámetros y variables asociadas, así como las restricciones que componen las condiciones del problema y además de una función objetivos de costos asociados al proceso de envió (transporte) (Quintero Ramirez, Omaña Silvestre, \& Sangerman-Joaquin, 2016).

Para ello se utilizó un modelo matemático (Hillier \& Lieberman, 2010) de "optimización" (Almada, 2017) sujeto a determinadas restricciones y variables. (López , Castro, \& Guerra, 2017) para definir una "ruta de origen - destino" (Taha, 2012, pág. 217) que se formula en (1).

$$
\text { Minimizar } \mathrm{z}=\frac{\sum}{\begin{array}{c}
\text { todos los arcos } \\
\text { definidos }(\mathrm{i}, \mathrm{j})
\end{array}} \text { CijXij }
$$

Dónde:

Xij $=$ Cantidad de flujo en el $\operatorname{arco}(i, j)$ $\{1$, si el arco $(i, j)$ esta en la ruta mas corta $\left\{\begin{array}{c}\text {, de lo contrario } \\ \text { d }\end{array}\right.$

Cij = Longitud del $\operatorname{arco}(i, j)$

Y dónde las restricciones representan la ecuación de la conservación del flujo en cada nodo que se indica en (2).

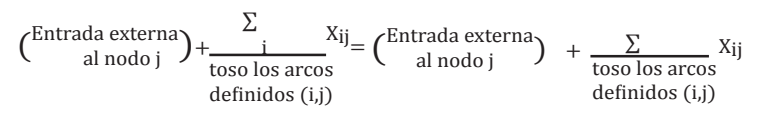

\section{Dónde:}

Flujo de entrada total = Flujo de salida total Y dónde, matemáticamente, esto se traduce así para el nodo j.

El modelo de programación lineal utilizado para el análisis se indica en (3). 


$$
\operatorname{Minimizar} \mathrm{Z}=\sum_{(\mathrm{i}=1)}^{\mathrm{n}} \sum_{(\mathrm{j}=1)}^{\mathrm{n}} \mathrm{dijX \textrm {Xj } ,} \quad \mathrm{dij}=\infty \text { para todas } \mathrm{i}=\mathrm{j}
$$

Dónde:

$\mathrm{Xij}=\left\{\begin{array}{c}1, \text { si se llega a la ciudad } \mathrm{j} \text { desde la ciudad } \mathrm{i} \\ \mathrm{o}, \text { de lo contrario. }\end{array}\right.$

Asumiendo que: dij =distancia de la ciudad i a la ciudad j

Sujeto a: $\sum_{j=1}^{n}$ Xij=1, $i=1,2 \ldots \ldots, n$

Con origen en $\mathrm{X}_{\mathrm{ij}}=(0,1)$ para todas las $\mathrm{i}$ y las $\mathrm{j}$.

Con estos instrumentos pueden ser cuantificados el costo de manejo de materiales, la relación lógica entre estaciones y tambíen la forma de cada área (Moreno, Alvarez, Noble, \& Lopez, 2014). La función objetivo buscó minimizar los costos asociados al transporte de los diferentes tipos de productos al Terminal desde la Refinería (Soto-De la Vega, Vidal-Vieira, \& Vitor-Toso, 2014). Considerando un Min Z que totaliza los Costos interfases generadas + Costos interfases adicionales + Costos penalización que se formula en 4 .

$$
\begin{aligned}
& \text { Min Z: } \sum_{\mathrm{j}=1}^{4}\left(\mathrm{CI}_{(\mathrm{j}) 1-2}+\mathrm{CI}_{(\mathrm{j}) 2-3}+\mathrm{CI}_{(\mathrm{j}) 3-4}+\mathrm{CI}_{(\mathrm{j}) 4-1}\right) \\
& \left.+\left(\mathrm{CI}_{(\mathrm{j}) \text { adicional }}\right)+\sum_{\mathrm{i}=1}^{4} \mathrm{CP}_{(\mathrm{j}) \mathrm{i}}\right)
\end{aligned}
$$

Dónde:

$\mathrm{CI}=$ Costo de interfase; $\mathrm{CP}=$ Costo de Penalización; J1= Compañía Copec; J2= Costo de Penalización; J3= Buque 1

J4= Buque 2; $1=$ Diesel; $2=$ Kerosene; 3 = Gasolina 93; 4= Gasolina $97 \mathrm{y}$, dónde $\mathrm{CI}($ adicional $)=$ Mezcla dependiente del producto postergado por falta de oferta.

En este caso la función del modelo está sujeto a la restricción de que las variables asociadas a las cantidades de producto a enviar deberán ser menor o igual a la capacidad de producción de la refinería relacionando "Función Objetivo y Restricciones" (Mejía, Hincapié, \& Gallego, 2015).

Finalmente, se planteó un modelo ficticio para la entrega de hasta 4 productos por cliente, cabe señalar que los clientes pueden alcanzar hasta 2 buques y 2 compañías, las cuales el total de entrega máxima es aproximadamente de 16 lotes para el envío. El contenido del oleoducto será Diesel, la secuencia estará condicionada al cliente, el orden de envío se determina para los diferentes clientes, considerando una óptima secuencia así como reducción de los costos por interface.

\section{Representación de Nodos}

En la figura 1, se representa la simplificación del problema, a través de una red de nodos, en la cual el software analiza cada ruta, comenzando por el nodo de inicio el cual pertenece al producto que permanece en la línea.

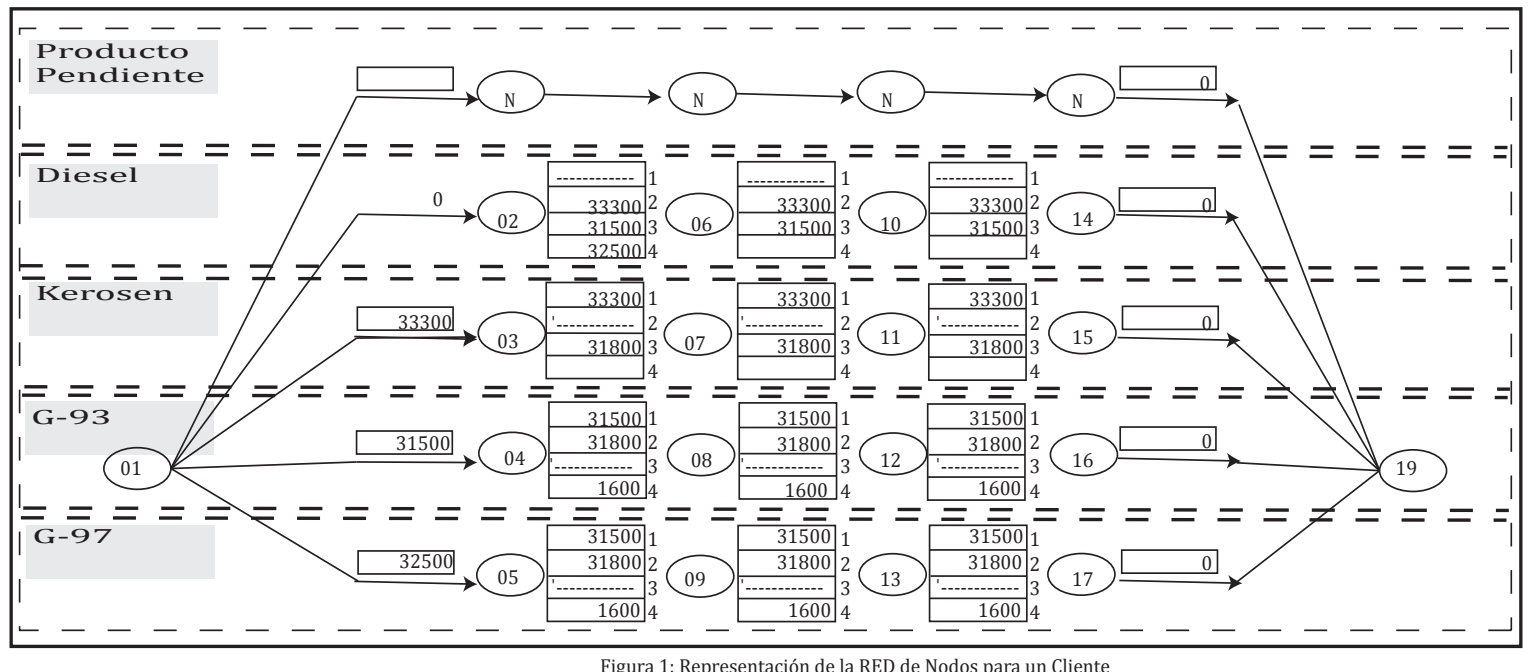

Se elaboró una planilla Excel que define producto inicial, inicio en el poliducto, demanda de los clientes, tipos de productos posibles y demanda. Esta planilla Excel es dinámica, ya que se generan los datos para cualquier situación de demanda, tanto de clientes como para la solicitud de productos. La gran variedad de posibilidades y casos en que la demanda de productos varia en volumen y en cantidad de productos solicitados, y hace que existan muchas alternativas de solución, es que se simplifico el problema omitiendo algunas condiciones.

\section{Costos Asociados a Interfaces}

Los costos asociados a las interfases, se obtienen de acuerdo al costo de interfase respecivo de cada mezcla de producto generada, considerando también la cantidad de mezcla que se produce para el tipo de interfase, el cual es mayor en el caso de ser contaminacion y ademas se considera que esta se envía a reproceso. 
Tabla 1 Costos de producción y de matriz de interfese

\begin{tabular}{|c|c|c|c|c|}
\hline \multicolumn{5}{|c|}{ Costos de Producción } \\
\hline Kerosene & \multicolumn{2}{|r|}{840,95} & & \\
\hline Gasolina 97 & \multicolumn{2}{|r|}{800,17} & & \\
\hline \multicolumn{5}{|c|}{ Matriz de Costos Asociados a los diferentes Interfases } \\
\hline & Diesel & Kerosene & Gasolina 93 & Gasolina 97 \\
\hline Gasolina 93 & 31500 & 31800 & o & 1600 \\
\hline Gasolina 97 & 32800 & 32800 & 1600 & o \\
\hline
\end{tabular}

El costo de pérdida por interfase, se estima en un $40 \%$ del costo de produccion de los productos mezclados de las diferentes corrientes del proceso en forma eficiente (Gordillo-Rios \& Quesada-Barrero, 2019), multiplicado por la cantidad de mezcla generada, dependiendo del tipo de interfase. Estos costos se representan en la siguiente tabla, como US\$ por interfase generada. Los valores de cada celda asociada al tipo de interfase entre las diferentes combinaciones, consideran el costo de produccion y la cantidad de mezcla generada.

\section{Resultados}

La investigación reaizada, de acuerdo al método de programación lineal (López, Castro, \& Guerra, 2017), y la utilización de programa WinQsb (Salazar, 2018) permite entre su variedad de aplicaciones, la de comparar las alternativas planteadas para el problema de distribución, los datos se debieron ajustar a esta opción, ya que realmente es una mezcla asociado a problemas denominado en la investigación de operaciones; "la ruta mas corta" y de "agente viajero", adaptando las datos en una matriz generada en Excel, que luego se importa al programa WinQsb. En base a lo planteado se puede obtener los siguientes resultados de alternativas viables:

\section{Alternativa 1}

Entrega individual a cada cliente, y entrega total de los productos mediante la utilización del software WinQSB éste es un paquete de herramientas bastante versátil que nos permite el análisis y la resolución de modelos: matemáticos, problemas administrativos, de producción, proyectos, inventarios, transporte, entre muchos otros. (Salazar, 2018). Además con lo planteado como la entrega de lotes para las compañías y a los buques anteriormente. Se obtuvo el siguiente resumen de secuencias óptimas para la solución al problema.

Tabla 2 Secuencias, ordenes de atención e interfase

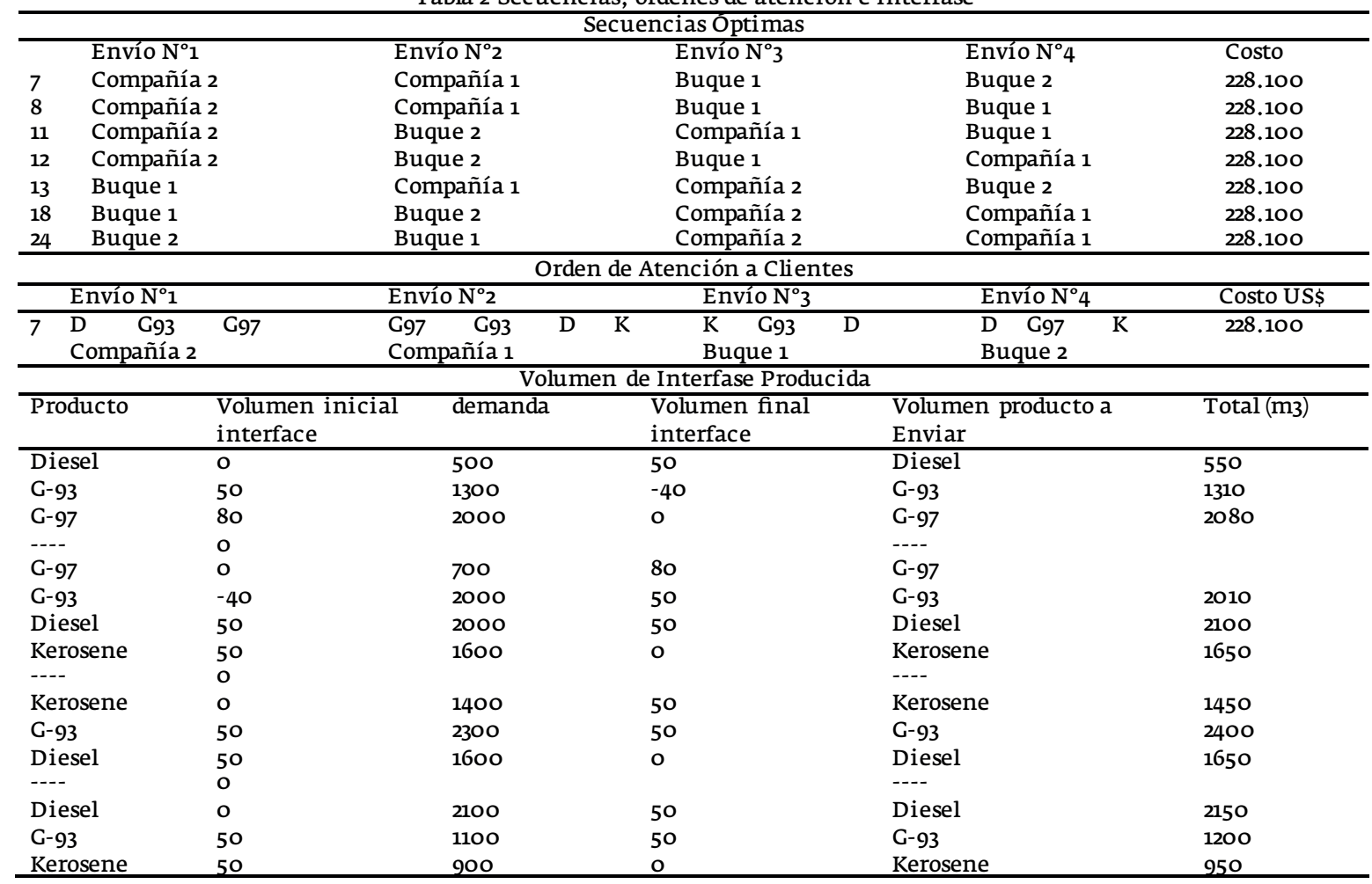


Dependiendo las prioridades de entrega, se eligió la opción más conveniente, en este caso se priorizara la entrega a las compañías y luego a los buques. Cómo solución óptima se determina la siguiente secuencia, como también el siguiente orden de atención a clientes: La planilla Excel, genera los volúmenes de producto a inyectar a la línea del poliducto, dependiendo de la secuencia obtenida en el software, el cual adiciona las cantidades de cada producto por efecto de la interface que se producirá en la mezcla.

\section{Alternativa $\mathrm{N}^{\circ} 2$}

Entrega agrupando a dos clientes, con entrega total de los productos, creando lotes de envío para estas dos demandas. Además de la penalización por demora en la entrega, y como resultado de una posible entrega parcial que se deberá adicionar interfaces debido a esta situación. Se obtienen 6 combinaciones para el agrupamiento entre los clientes, las cuales 3 son las principales y las otras 3 son repeticiones de las anteriores, debido al ordenamiento inverso que tiene un mismo valor para el costo de espera. Los resultados y opciones obtenidos se muestran en la siguiente Tabla 3:

Tabla 3 Opciones de la Alternativa 2

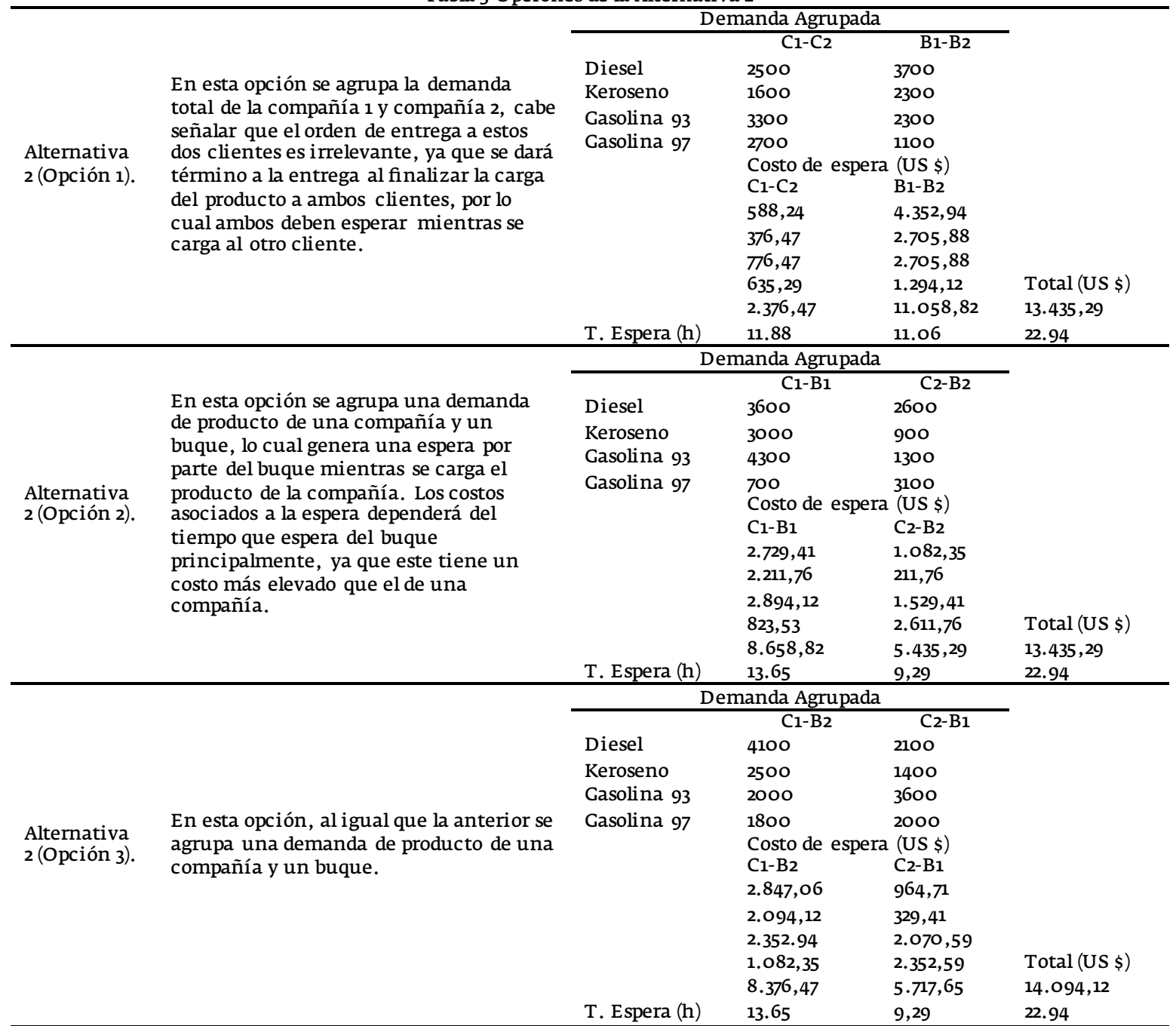

Los datos obtenidos del costo total en espera, se incluyen en la matriz a generar en el programa Excel, este valor se vincula al costo asociado a cada alternativa en su respectiva celda, que está ubicada al final de la ruta de cada opción, posteriormente se analizo la matriz en el Programa Win QSB. Se observa que en la opción 3, como también el la 6 que es el orden inverso de entrega entre los dos clientes del grupo tienen un menor costo.
Para las soluciones obtenidas, se estableció que el envío se debe realizar para la compañía 1 y buque 2 como primer grupo de clientes, y posteriormente a la compañía 2 y buque 1 como segundo lote de envío.

En siguiente tabla se definió la cantidad de producto a inyectar a la línea del poliducto, enviando las cantidades definidas de acuerdo a la secuencia obtenida en el programa WinQsb, sumando a estas cantidades las 
interfaces respectivas a la mezcla generada. Para efecto de comparación entre las alternativas, se plantea que las condiciones de entrega de productos a los respectivos clientes tiene condicionado la estadía de los buques así como la espera de las compañías, para el caso de alguna secuencia, como también la prioridad de entrega que arroje el programa como solución óptima al problema demandado.

Los resultados obtenidos en la segunda alternativa tiene un costo mucho menor que la primera alternativa, ya que para el agrupamiento de demanda de productos entre los clientes, se reducen las inter ases a casi a la mitad.

Cabe señalar que la primera alternativa, se realiza el envío de productos a cada cliente por separado, definiendo así la secuencia de entrega de productos a cada cliente, es así como el costo para evaluar esta alternativa se consideró solamente el costo asociado a las inter ases, no así en la segunda alternativa, en que además considera el costo de espera de los clientes al realizar la entrega en lotes, agrupando las dos demandas respectivas, pero se ahorrarán las cantidades de inter ases generadas al agrupar.

Tabla 4 Análisis, envíos, mezclas y resultados

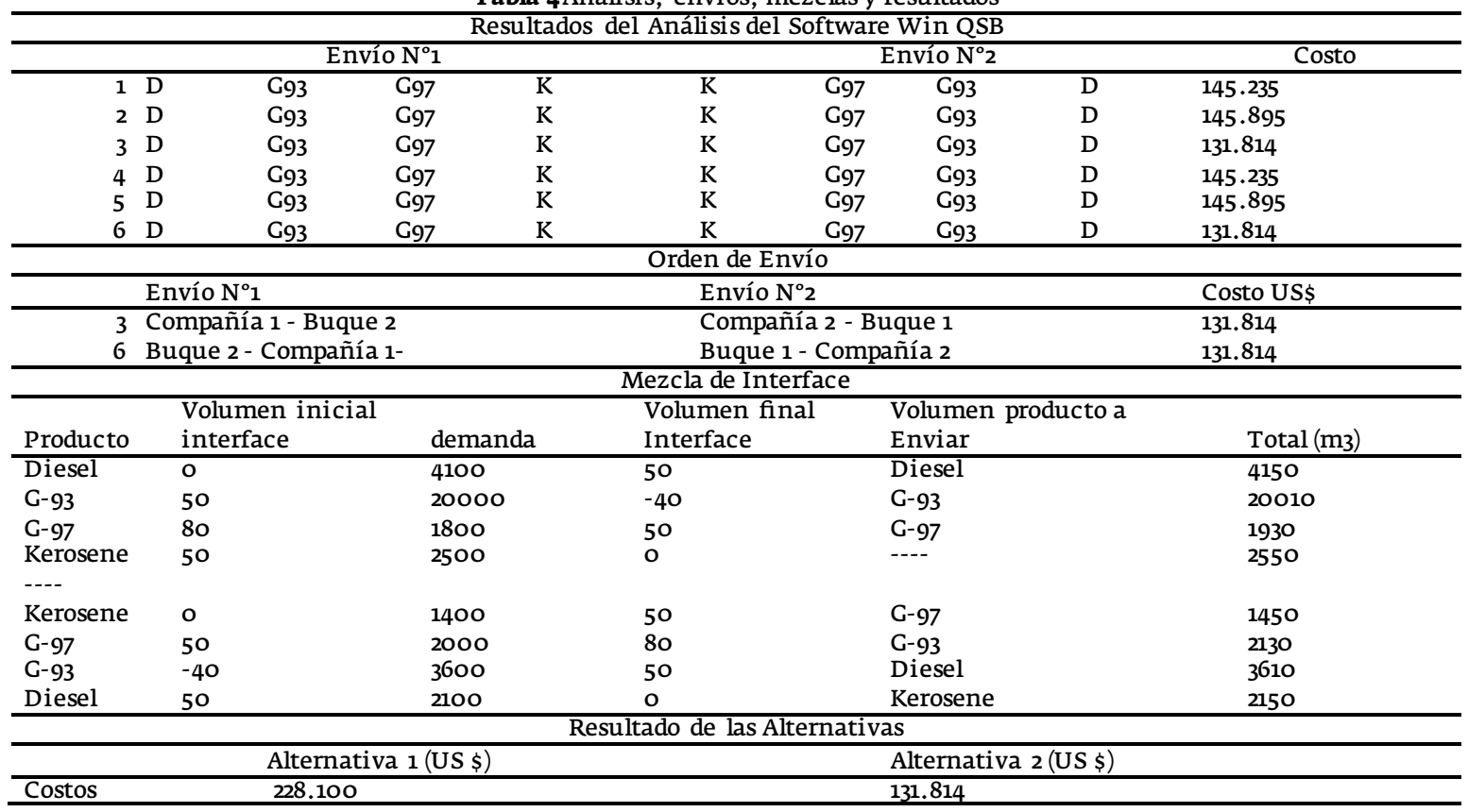

Conclusiones

De acuerdo al analisis de la optimización de secuencia se concluye que la diferencia de costos del modelo planteado es por la reducción de interfases, esto referido a los costos asociados a pérdidas por la generación de interfases, pese a que las condiciones de servicio solo se realiza como una entrega total de los productos requeridos por cada cliente.

La evaluación de la alternativa de entregar los productos agrupando dos clientes, enviando lotes de productos demandados por los respectivos clientes, generó un ahorro de 96.286 dólares, solo en un ciclo de envío a los 4 clientes, conforme la demanda establecida para el ejemplo planteado.

El ahorro está condicionado a que los clientes están dispuestos a postergar o a esperar la emtrega de sus productos por un pago asociado a esta espera, como una penalización de entrega a la refinería. Este costo se estimo solo para analizar la segunda alternativa.

La solución que mejora la distribución y reduce los costos entre las alternativas planteadas, deriva principalmente de la reduc- ción de interfaces al agrupar lotes de envío de productos, y en menor medida, del costo asociado al tiempo de espera. Finalmente, se propone realizar el envío y distribución de los productos demandados agrupados, ya que entregara la mejor secuencia de inyección de los diferentes productos a la línea.

\section{Referencias}

Almada, M. L. (2017). Transporte de Hidrocarburos por ductos sin transbordos. Universidad de la Defensa Nacional, Facultad de Ciencias de la administración, Buenos Aires, argentina. Arango Serna, M. D., Zapata Cortes, J. A., \& Gomez Montoya, R. A. (Julio-Diciembre de 2010). Estrategia en la cadena de Suministro para el distrito minero de Aragá. Boletin de Ciencias de la Tierra(28).

Bernal, C. A. (2010). Metodologia de la Investigacion. Colombia: Pearson.

Cafaro, D. (2009). Programacion óptima de operaciones en sitemas de transporte de cobustibles a traves de poliductos. Santa Fe: Tesis Doctor en tecnologia Quimica.

Chase, R. B., Jacobs, F., \& Aquilano, N. J. (2009). Administración de Operación. Producción y Cadena de Suministros. México, México D.F., México: Mc Graww Hill.

De la Hoz, E., Vélez, J., \& Lopez, L. (2017). Modelo de Programación Lineal para logistica Inversa en el sector Polipropileno. Información Tecnológica, 28(5). Feitó Cespón, M., Cespón Castro, R. , \& Rubio Rodriguez, M. 
a. (Enero de 2016). Modelos de Optimización para el diseño sostenible de cadenas de suministros de reciclaje de multiples productos. Revista Chilena de Ingeniería, 24(1).

Ferrer, I., Cones, A., \& De los Santos, M. (2004). Modelamiento del Transporte de Distribución Mediante Programación Lineal Entera. Información Tecnológica, 15(4).

Gallardo, R. A. (2011). Tesis Minimizacion de la tardanza Total de una maquina con tiempos de preparacion dependientes de la secuencia mediante de Modelos de programacion Entera Mixta. Concepción: Universidad del Bio Bio.

Gamez-Albón, H. M., Mejía-Argueta, C., \& León Espinoza de los Monteros, R. A. (Diciembre de 2017). diseñode una Red de Distribución a través de un Modelo de Optimización considerado agotados. revista Chile de Ingeniería, 25(4).

Garcia Sánchez, A. (2007). Tesis Programacion del transporte de Hidrocarburos por oleoducos mediante la combinación de técnicas metaheurísticas y simulacion. Madrid: Universidad Politécnica de Madrid.

Gordillo-Rios, G., \& Quesada-Barrero, L. (Enero-Abril de 2019). Diseño de una Tobera Mezcladora para un Tan que de Gasolina. Revista Cubana de Quimica, 31(1).

Hillier, F. S., \& Lieberman, G. J. (2010). Introducción a la Investigación de Operaciones. México, México D.F., México: Mc.Graw Hill.

López Calvajar, G., Castro Perdomo, N., \& Guerra, O. (Enero-Marzo de 2017). Optimización del Plan de Producción. Estudio de Caso crpintería de Aluminio. Universidad y Sociedad, 9(1).

Mejía Sülamilla, A. M., Hincapié Isaza, R. A., \& Gallego Rendón, R. A. (Enero-marzo de 2015). Planeación öptima de Sistemas de Distribución considerando Múltiples Objetivos: Costo de Inversión, Confiabilidad y Pérdidas Técnicas. Tecnora, 19(43).

Moreno, A., Alvarez, A., Noble, V., \& Lopez, J. (Julio-Diciembre de 2014). Optimización Multiobjetivo del Problema de Distribución de Planta: Un nuevo Modelo Matemático. Ingeniería y Competitividad, 16(2).

Noguera Polania, J., Robles Aljarin, c., \& Guillot Fulla, J. (Enero de 2015). Control predictivo para Operar una Red de Bombas centrifugas en un Sistema Serial de Poliductos. Revista Chilena de Ingeniería, 23(1).
Orjuela-Castro, J. A., Suarez-Camelo, N., \& Chinchilla-Ospin, Y. I. (Julio-Diciembre de 2016). Costos Logísticos y Metodología para el Costeo en Cadenas de Suministros: Una revisión de la Literatuta. Cuadernos de Contabilidad, 17(44).

Peña Orozco, D. L., Urueña Villamil, J. F. \& Conzález Valencia, L. A. (2016). Diseño de una Red Logistica para una Comercializadora ferretera en el centro del Valle del Cauca. Entramado, 12(1).

Quintero Ramirez, J. M., Omaña Silvestre, J. M., \& Sangerman-Joaquin, D. M. (2016). Modelo de Transporte para la distribución de Guayaba( psidium Guayava L.) en México. Revista Mexicana de Ciencias Agrícolas, 7(6).

Quesada Ibargüen, V. M., \& Vergara Schmalbach , J. C. (2006). Manual Análisi cuantitativo con WINQAS. Cartagena: Universidad de Cartagena.

Salazar, B. (2018). Ingeniería Industrial Online Recuperado el marzo de 2019, de Ingeniería Industrial: http//: www.ingenieríaindustrialonline.com

Soto-De la Vega, D., Vidal-Vieira, J. G. \& Vitor-Toso, E. A. (Marzo-Abril de 2014). metodología Para Localización de Centros de Distribución a Través de Análisis Multicriterio y Optimización. DYNA Fac. Nac. Minas, 81(184).

Taha, H. A. (2012). Investigación de operaciones. Estado de Mexico: Pearson Educación.

Toro Ocampo, E., Dominguez Castaño, A., \& Zuluaga Escobar, A. (Enero-Junio de 2016). Desempeño de las Técnicas de Agrupamiento para resolver el problema de ruteo con multiples depósitos. tecnologicas, 29(36).

Torres, L., Verde, C., Carrera, R., \& Cayetano, R. (Julio-Agosto de 2014). Algoritmo de diagnóstico para fallas en Ductos. Tecnología y Ciencia del Agua, 5(4).

Vinajera-Zamora, A., Maruro-Delgado, F., \& Ruiz-Morales, M. (Septiembre de 2017). Método para calcular el valor agregado en Cadenas de Suministros de Productos Electromecanicos. Revista Chilena de Ingeniería, 25(3), 2. 